\title{
EFFECT OF KINETIC PARAMETERS ON GROWTH OF ONE-DIMENSIONAL TIN OXIDE NANOSTRUCTURES
}

\author{
M. SALEHI \\ Department of Materials Science and Engineering, Sharif University of Technology \\ P.O. Box 11365-9466, Tehran, Iran \\ msalehi1385@yahoo.com \\ S.K. SADRNEZHAAD* \\ Department of Materials Science and Engineering, Sharif University of Technology \\ P.O. Box 11365-9466, Tehran, Iran \\ sadrnezh@sharif.edu
}

\begin{abstract}
One-dimensional tin oxide nanostructures were synthesized by carbothermal reduction process. Results showed that using Au as catalyst on Si wafer and shorter distance from source materials lead to higher nucleation and growth rate. It was understood that $\mathrm{SnO}_{2}$ to carbon mixing ratio of 1:4 is appropriate. $\mathrm{SnO}_{2}$ nanostructures were grown by VLS mechanism.
\end{abstract}

Keywords: $\mathrm{SnO}_{2}$; One-dimensional nanostructure; metallic catalyst.

\section{Introduction}

Tin dioxide $\left(\mathrm{SnO}_{2}\right)$ is an important semiconductor with a wide band gap. It has potential applications in optoelectronic devices including flat panel displays and solar energy cells. This material has been extensively used for gas sensors. ${ }^{1-3}$

Since one dimensional (1D) nanostructures such as nanowires, nanotubes, nanobelts, nanorods and nanoribbons have high surface to volume ratio, synthesis of these morphologies shows increasing interest. Up to now, $\mathrm{SnO}_{2} 1 \mathrm{D}$ nanostructures have been synthesized by different methods like hydrothermal treatment, thermal evaporation, oxidation techniques and spray pyrolysis. ${ }^{4-6}$

Herein, we synthesized $\mathrm{SnO}_{2}$ 1D nanostructures by the simple carbothermal reduction method, successfully. Effect of different parameters including absence or presence of catalyst, ratio of the source materials, the substrate- source materials distance and flow of argon were investigated.

\footnotetext{
*Corresponding author. Tel: +98 2166165215.

Email addresses: $\underline{\text { sadrnezh@ } @ \text { sharif.edu and sadrnezh@yahoo.com }}$
} 


\section{Experimental Procedure}

Silicon wafers were employed as substrate. Substrates were cleaned and some of them were coated with Au by sputtering at $10^{-3}$ torr and $17 \mathrm{~mA}$ electric current. $\mathrm{SnO}_{2}$ powder was thoroughly mixed with activated carbon with $1: 1,1: 4$ and 1:8 ratios as the source materials. Source materials and the substrates were placed at the center of the furnace and at 5 and $7 \mathrm{~cm}$ from the center of furnace, respectively.

Source materials were heated at $950^{\circ} \mathrm{C}$ for $1 \mathrm{hr}$ and then cooled down to the room temperature. The as-synthesized products were characterized by X-ray diffraction (XRD) and scanning electron microscope (SEM, British, 1990, Sterio Scan 360 Leica/ Cambridge).

\section{Results and Discussion}

Figs. 1-3 indicate morphologies of the $\mathrm{SnO}_{2}$ nanostructures synthesized at $950^{\circ} \mathrm{C}$ for $1 \mathrm{hr}$. At the mixing ratio of $1: 1$, only a few nanowires could be grown. With the ratio of $1: 4$, the density and the length of the nanostructures remarkably increased.

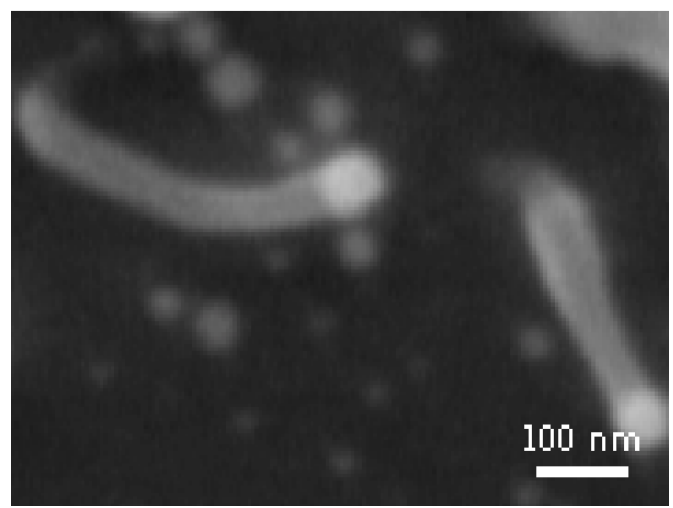

Fig. 1. SEM image of $\mathrm{SnO}_{2}$ nanostructures grown with $\mathrm{SnO}_{2}$ : C ratio of 1:1.

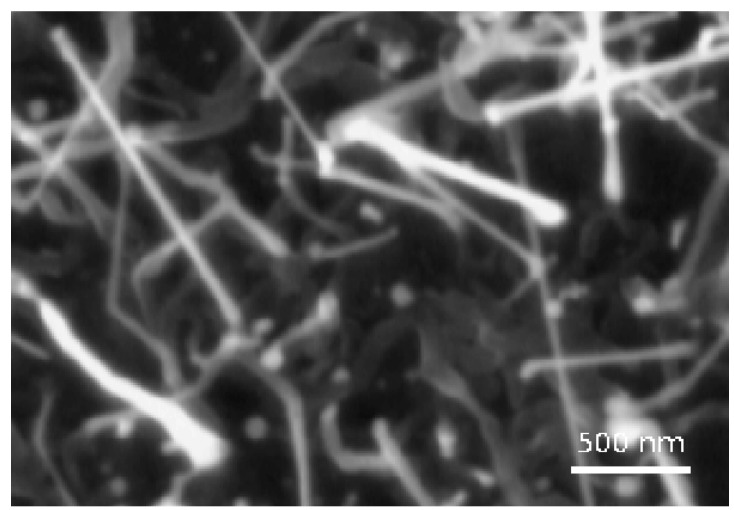

Fig. 2. SEM image of $\mathrm{SnO}_{2}$ nanostructures grown at $\mathrm{SnO}_{2}$ : C ratio of 1:4. 


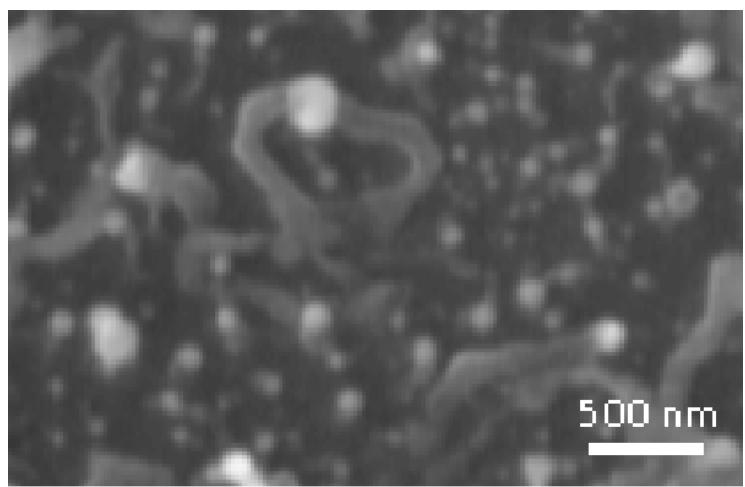

Fig. 3. SEM image of $\mathrm{SnO}_{2}$ nanostructures grown at $\mathrm{SnO}_{2}: \mathrm{C}$ ratio of 1:8.

$\mathrm{SnO}_{2}$ to carbon ratio of 1:8 was expected to lead to further nucleation and growth as compared to other ratios examined. It did not, however, occur perhaps due to the excess carbon atoms that could surround the $\mathrm{SnO}_{2}$ particles and prevented from reaction with $\mathrm{CO}$. The ratio of 1:4 was, hence, selected as the appropriate mixing ratio and was used for studying the tests. The diameter and the length of the 1D nanostructures synthesized were within 30-100 $\mathrm{nm}$ and several micrometers ranges, respectively.

Fig. 4. shows the XRD pattern of the synthesized sample with $\mathrm{SnO}_{2}$ powder to activated carbon mixing ratio of 1:4. The diffraction peaks are indexed as $\mathrm{SnO}_{2}$ (JCPDS. File No. 1-0657) and Au (JCPDS. File No.1-1172).

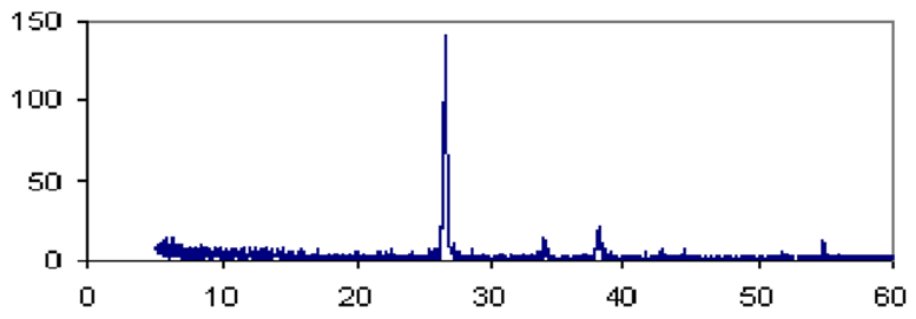

Fig. 4. XRD pattern of the product.

The presence of the catalyst affects on the morphology of the fabricated $\mathrm{SnO}_{2}$ nanostructures. Fig. 5 shows the SEM image of the nanostructures grown on the silicon without $\mathrm{Au}$. It can be seen that there are only few 1D nanostructures on the bare substrate. From Fig. 2, it is observed that nucleation and growth of nanostructures increase on the Au-coated silicon. 


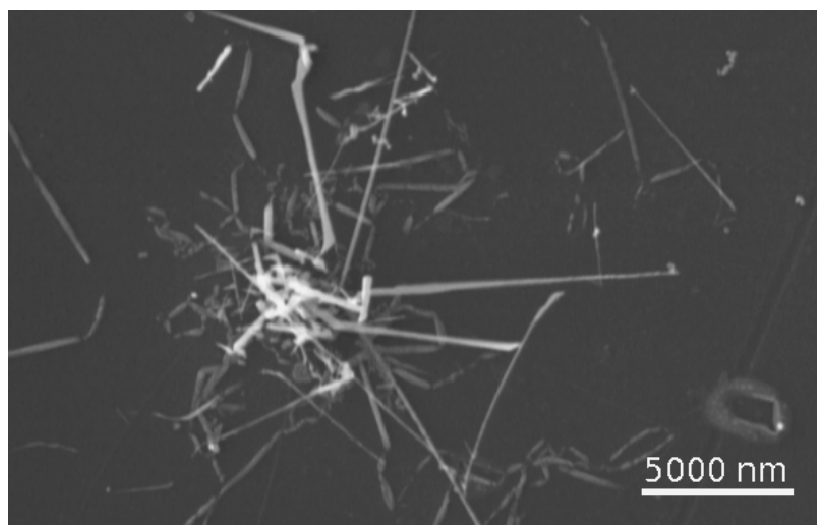

Fig. 5. SEM image of $\mathrm{SnO}_{2}$ nanostructures grown on the bare substrate.

Based on the SEM images, growth of the nanostructures follows a Vapor-Liquid-Solid (VLS) mechanism. The essence of VLS mechanism is the presence of the liquid phase. Usually, a noble metal such as Au is used as a catalyst. At high temperatures, Au thin film will coalesce and form spherical particles on $\mathrm{Si}$ wafer. The $\mathrm{Au}$ particles have a higher sticking coefficient and is a preferred site for deposition of the tin- bearing gas species produced from the carbothermal reaction. With continuous adsorption, $\mathrm{Au} / \mathrm{Sn}$ alloy supersaturates and growth of $\mathrm{SnO}_{2}$ occurs. ${ }^{7-10}$

In this work, there are particles at the tip of the 1D nanostructures which confirm the VLS growth mechanism. In order to find the relationship between the temperature difference and the amount of the growth of the nanostructures, the Au-coated substrates were placed at different distances from the center of the furnace as shown in Fig. 6.

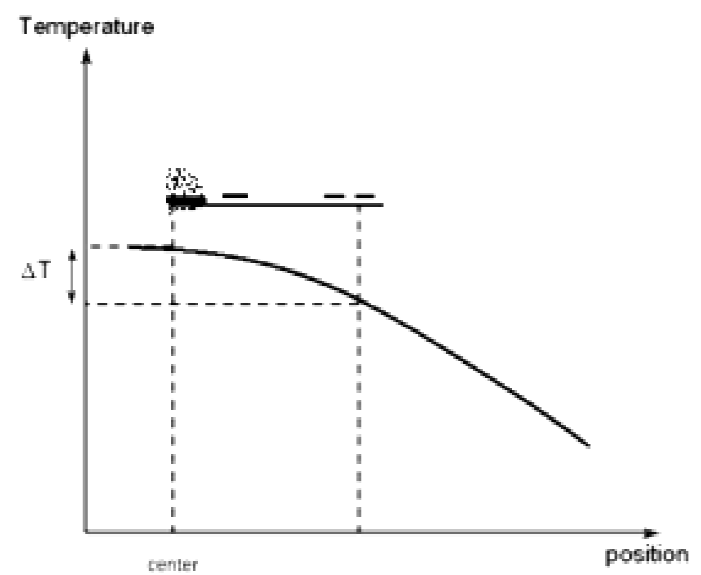

Fig. 6. Schematic illustration of temperature against distance from source materials. 
Figs. 7 and 8 show the SEM images of the samples located at distances about 5 and 7 $\mathrm{cm}$ from the source materials. It can be seen that the amount of the nanostructures produced decrease with the distance from the source materials. According to the Fick's first law (Eq. 1):

$$
\mathrm{J}=-\mathrm{D}(\mathrm{dN} / \mathrm{dX})
$$

concentration of the tin - bearing gas species slow at the farther distance from the source materials. $^{11}$

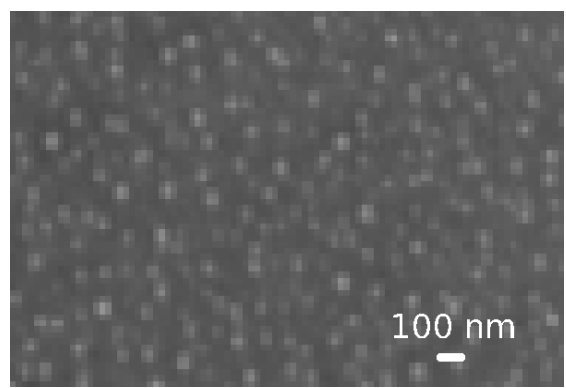

Fig. 7. SEM image of the nanostructures grown on the substrate at distance $5 \mathrm{~cm}$ away from source materials.

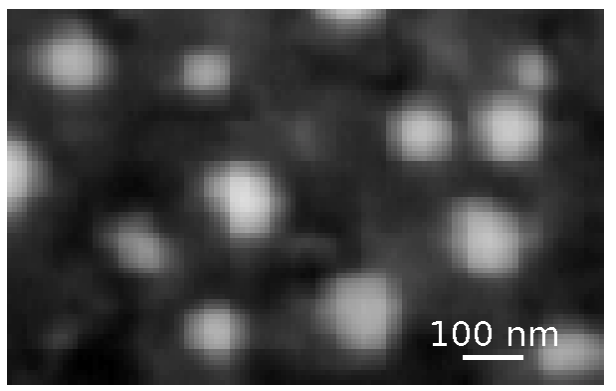

Fig. 8. SEM image of the nanostructures grown on the substrate $7 \mathrm{~cm}$ away from source materials.

With the experimental conditions of this research, under the flow of Ar, more tin oxide nanostructures appeared. In fact, the flow of Ar caused the tin - bearing gas species transfer to the substrate (not shown here).

\section{Conclusions}

One-dimensional tin oxide nanostructures were successfully synthesized by carbothermal reduction process. Using Au significantly affected the nucleation and growth of the tin oxide nanostructures. The rate of growth increased with increasing of the $\mathrm{SnO}_{2}$ to carbon 
ratio to $1: 4$ and decreased by increasing the ratio to $1: 8$. The growth rate decreased with increasing of the distance from the source materials.

\section{References}

1. M.O. Orlandi, A.J. Ramirez, E.R. Leite and E. Longo, Crystal growth \& design, 8, 1067, (2008).

2. H.W. Kim, S.H. Shim, Vacuum, 82, 1395, (2008).

3. Z. Rong, Z. Wei Pan and Z. Wang, J. Am. Chem. Soc., 124, 8673, (2002).

4. P.G. Li, M. Lei and W.H. Tang, et al, Journal of Alloys and compounds, doi:10.1016/j. jillcom, (2008).

5. X. Wu, J. Sui, W. Cai and F. Qu, Materials chemistry and Physics, 112, 325, (2008).

6. Z. Huang, C. Chai, Materials letters, 61, 5113, (2007).

7. D. Zhao, C. Andreazza, et al, Chemical Physics Letters, 399, (2004).

8. Y. Su, X. Liang and, S. Li, Y, et al. Mterials letters, 62, 1011, (2008).

9. M. Lin, T. Sudhirajan, and C. Boothroyd, et al, Chemical physics letters, 400, 175, (2004).

10. C. Li, G. Fang, and Q. Fu, et al, Journal of nanoscience and nanotechnology, 6, 1473, (2006).

11. D.H. Fan, Y. Bai, Journal of crystal growth, doi: 10.1016/j. jcrysgro, (2009). 